\title{
THE NUMERICALLY GENERATED SERIES EXPANSIONS FOR SPIN-LATTICE SYSTEMS
}

\author{
G. MUSIAE* \\ Computational Physics Division, Institute of Physics, A. Mickiewicz University \\ Umultowska 85, 61-614 Poznań, Poland
}

The numerically generated series expansions complementary to widely used computer simulations of the Monte Carlo type, are presented. The effective algorithm for generation of graphs, which constitute the basis of series expansions for spin-lattice systems, is introduced. The use of the numerically generated series expansions to calculate the free energy for the eight-vertex model is explained and discussed in detail.

PACS numbers: $02.60 .-\mathrm{x}, 75.10 . \mathrm{Hk}, 75.40 . \mathrm{Cx}$

\section{Introduction}

Series expansions (SE) are widely used in physics and astronomy. Description of physical phenomena in which interactions between degrees of freedom of the system completely change the character of the solution, requires derivation of a large number of terms in such expansions. Classification of the contributions of higher order terms leads naturally to a description in terms of linear graphs ${ }^{\dagger}$ (for the review see [1]), in which bonds correspond to interactions. SE have attracted particular attention in statistical mechanics, and especially the low- and high-temperature expansions have led to important progress in the theory of phase transitions and critical behaviour of spin-lattice systems. Investigation of complex models of these systems is usually carried out by computer simulation methods of the Monte Carlo type, whose results should be confirmed with the aid of other complementary methods. which are SE, provided that they contain a sufficient amount of terms.

Our aim is to prepare a numerical procedure of effective generation of arbitrary long $\mathrm{SE}$ to analyse a wide class of spin-lattice systems. We will present this procedure calculating the free energy for the eight-vertex model, as it represents a wide class of such systems.

\footnotetext{
*e-mail: gmusial@zawrat.amu.edu.pl

$\dagger$ A linear graph is a collection of $k$ points (called vertices) with $i$ bonds between certain pairs of vertices and one pair of vertices connected by one bond.
} 


\section{Calculation of the free energy}

The numerically generated SE will be discussed on the example of the eight-vertex model on a simple cubic lattice, for which the zero-field effective Hamiltonian is of the form

$$
-\beta H=K_{1} \sum_{\langle i, j\rangle} \sigma_{i} \sigma_{j}+K_{2} \sum_{\langle i, j\rangle} \tau_{i} \tau_{j}+K_{4} \sum_{\langle i, j\rangle} \sigma_{i} \tau_{i} \sigma_{j} \tau_{j},
$$

where the angle brackets denote summation over nearest neighbors, $\beta=1 / k_{\mathrm{B}} T$, $k_{\mathrm{B}}$ is the Boltzmann constant, $T$ - the temperature, $\sigma_{i}, \tau_{i}= \pm 1$ are the spin variables residing at the same site of the lattice, $K_{1}$ and $K_{2}$ are couplings between two neighboring $\sigma$ spins and $\tau$ spins, respectively, whereas $K_{4}$ is the coupling between all four spins residing at two neighboring sites of the lattice. This Hamiltonian has a general form and allows concluding about a wide class of classical models; e.g., for $K_{2}=K_{1}$ this model reduces to the Ashkin-Teller (AT) model.

As an illustration of calculations of thermodynamic functions we explain how to calculate the reduced free energy of the model

$$
f=-\frac{F}{N k_{\mathrm{B}} T}=\frac{1}{N} \ln Z,
$$

using the SE numerical technique. Here $F$ is the free energy and $N$ is the number of sites in the lattice. The most important quantity which allows us to calculate the free energy (and the other thermodynamic functions) is the partition function $Z=\operatorname{Tr} \mathrm{e}^{-\beta H}$. For further calculations we perform the transformation $\mathrm{e}^{K_{1} \sigma_{i} \sigma_{j}+K_{2} \tau_{i} \tau_{j}+K_{4} \sigma_{i} \tau_{i} \sigma_{j} \tau_{j}}=a+b \sigma_{i} \sigma_{j}+c \tau_{i} \tau_{j}+d \sigma_{i} \tau_{i} \sigma_{j} \tau_{j}$ and we use the fact that $\sigma_{i}, \tau_{i}= \pm 1$, which allows us to write down all nonequivalent equations (there are 4 such equations) and to obtain the expressions for the constants $a, b, c, d$ in terms of the couplings $K_{1}, K_{2}, K_{4}$, e.g. $a=\operatorname{ch} K_{1} \operatorname{ch} K_{2} \operatorname{ch} K_{4}+\operatorname{sh} K_{1} \operatorname{sh} K_{2} \operatorname{sh} K_{4}$. Thus, we have

$$
Z=\operatorname{Tr} a^{N z / 2} \prod_{\langle i, j\rangle}\left(1+v_{1} \sigma_{i} \sigma_{j}+v_{2} \tau_{i} \tau_{j}+v_{4} \sigma_{i} \tau_{i} \sigma_{j} \tau_{j}\right),
$$

where $v_{1}=b / a, v_{2}=c / a, v_{4}=d / a$.

After multiplication of all terms in the product in Eq. (3) we obtain a sum of other terms that contain some powers of spins, $v_{1}, v_{2}$ and $v_{4}$. The trace can be calculated separately for every term and then only these terms in which spins appear in even powers give non-zero contribution to the sum. This means that spins contribute a factor 1 in every non-vanishing term. We can order terms with increasing $s$ ( $s$ is the sum of powers at $v_{1}, v_{2}$, and $v_{4}$ ) and truncate the sum rejecting terms with $s>s_{\max }$, which should be chosen big enough to obtain the desired precision of computation.

Such an ordering of terms leads naturally to a description in terms of linear graphs in which bonds correspond to these interactions between spins which are specified in the Hamiltonian. The fact that spins appear only with even powers means that only closed graphs give non-zero contribution to the free energy.

Using graph representation we can write the partition function in the form

$$
Z=a^{N z / 2} 2^{2 N}\left[1+\sum_{g} N^{i(g)} l(g) v_{1}^{j(g)} v_{2}^{n(g)} v_{4}^{m(g)}\right],
$$


where summation runs over all non-isomorphic graphs $g$ in increasing order ${ }^{\ddagger}$, $i, j, n, m$ are integer numbers and $l$ is the lattice constant of a graph ${ }^{\S}$. As discussed above after Eq. (3), we sum over all graphs with orders lower or equal to $s_{\max }$. In Sec. 3 we propose the effective algorithm of generation of graphs and we explain how to obtain the values of the integers $l, j, n, m$.

Following Eqs. (2) and (4) and expanding In we get the final expression for computation of the reduced partition function:

$$
f=2 \ln 2+\frac{z}{2} \ln a+\sum_{g} l(g) v_{1}^{j(g)} v_{2}^{n(g)} v_{4}^{m(g)} .
$$

\section{The algorithm of calculations}

We propose here the algorithm which generates all closed connected linear graphs of the $i$-th order, with $i \leq s_{\max }$. We generate graphs starting from polygons which are the simplest graphs and simultaneously they are the starting point for further calculations. Next graphs are obtained by choosing in the starting graph of all possible pairs of not directly connected vertices and next by consequent projections of the vertex ${ }^{\text {I }}$ of a higher number (which disappears) onto the other one in the chosen pairs. At the same time the chosen pairs of vertices are connected by additional bonds forming other new graphs.

An important matter is the way of labelling of graphs. We assign the label to each graph using canonical labelling [1] and in such a way we generate only topologically different graphs. Two graphs which can be put into 1 to 1 correspondence, so whose vertices and bonds correspond, are isomorphic and should get the same label.

The label of every graph obtained as a result of projection of vertices onto themselves is added at the end of the set of generated graphs, provided it has not been generated earlier. The algorithm ends the calculations when all graphs from the set have undergone the projection procedure and from the last one no new graph arose. The resulting set of graphs is arranged according to increasing orders of the graphs. It is worth noting that up to this point, the procedure proposed here is independent of the choice of the model and the lattice geometry.

In the next step graphs are coloured, i.e. a specific spin-spin interaction together with a coupling constant is assigned to every bond in the graph, which is connected with the choice of a specific model. This assignment has to be done in every possible way to simulate every possible configuration of the system in SE. As far as the eight-vertex model is considered, a graph contains $j$ bonds of $v_{1}$ type, $n$ bonds of $v_{2}$ type and $m$ bonds of $v_{4}$ type. Here also graphs with odd number of bonds joined to a vertex are possible to get coloured here, which means that we have many more graphs when compared to those used for the Ising model.

$\ddagger$ The order $s$ of the graph is the number of bonds that it contains. One interaction introduces one bond in the graphs, here $s=j+n+m$ because $i$-th interaction introduces also $v_{i}$ to the term that is represented by the graph.

$\hat{s}$ The lattice constant of a graph is a number of nonequivalent puttings around 1 site of the lattice divided by a symmetry factor of the graph.

IProjection of one vertex onto another physically means that they overlap. 
After this stage of calculations, certain connected graphs are rejected, particularly in more complicated models. Therefore, only after the graphs have been coloured, all non-connected graphs should be constructed as combinations of connected graphs of lower orders whose sum is the order of the complex graph.

After all graphs are completed and coloured, the lattice constants are calculated for them as defined above (for details see e.g. [1]) which is related to the choice of a specific geometry of the lattice.

\section{Conclusions}

The basic elements for numerically generated SE are the algorithms for graphs generation and colouring. The proposed algorithms work effectively but the user has to have at his disposal enough space of operational memory in a computer with 64-bit processor (more than $2 \mathrm{~GB}$ to obtain the complete set of about $10^{5}$ graphs up to 15 -th order when the maximum number of bonds at a graph vertex is limited to 6). Another way of comparing of the new generated graphs with graphs which have been obtained before and which are stored on hard disk is too time consuming to generate the complete set of graphs of order greater than 11 in the realistic time.

As an example of a thermodynamic function the free energy in the series expansion has been considered. Analogously, starting from the partition function, other thermodynamic functions can be calculated.

Long SE are necessary to analyze the $3 \mathrm{D}$ version of many spin-lattice systems with sufficient accuracy. Using our experience in studying the 2D AT model [2] and the numerical procedure presented here we plan to consider the 3D AT model, whose phase diagram is still not fully resolved and rises many interesting points like e.g. the character of transition from para- to antiferromagnetic phase [3].

The outlined subroutines enabled us to construct and colour graphs up to 15 order (higher orders are also possible) which ensures sufficient precision of the $\mathrm{SE}$ results e.g. for the $3 \mathrm{D}$ AT model.

\section{Acknowledgments}

The author wishes to thank Prof. G. Kamieniarz and Prof. J. Rogiers for valuable discussions as well as the Poznan Supercomputing and Networking Center for the access to SGI Power Challenge XL supercomputer. Financial support from the Committee for Scientific Research within the grant 2P03B 07516 is also acknowledged.

\section{References}

[1] C. Domb, in: Phase Transitions and Critical Phenomena, Eds. C. Domb, M.S. Green, Vol. 3, Academic, New York 1974, p. 1; J.L. Martin, ibid., p. 97.

[2] P. Pawlicki, G. Musiał, G. Kamieniarz, J. Rogiers, Physica A 242, 281 (1997); P. Pawlicki, G. Kamieniarz, L. Dębski, ibid., p. 290.

[3] R.V. Ditzian, J.R. Banavar, G.S. Grest, L.P. Kadanoff, Phys. Rev. B 22, 2542 (1980). 